Abstract EPV039/\#175 Table 1 Individual insurance status, Hospital payer mix and associated mortality among cervical cancer patients

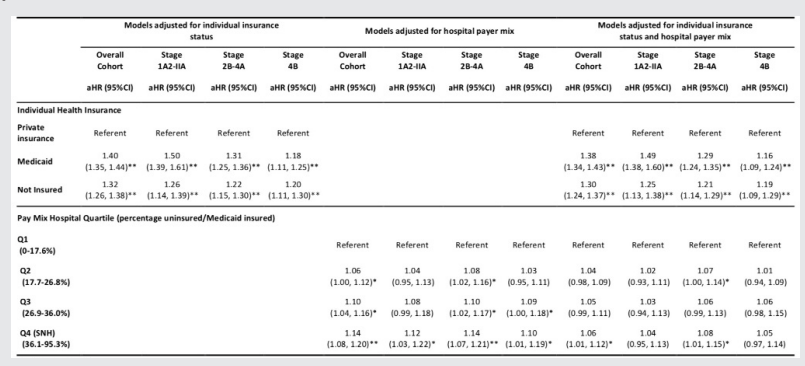

${ }^{*} \mathrm{P}<0.05,{ }^{*} \mathrm{P}<0.001$.

Marginal cox proportional hazards models adjusted for hospital clustering, patient's age, race, year of diagnosis, zip code median house income quartile, charlson comorbidity score, cancer substage, histology, grade and tumor size. Values reported as hazard ratios with $95 \%$ confidence interval.

Change of AIC (Akaike information criterion) in multivariable model omitting individual insurance $=731$; change of AIC in multivariable model omitting individual insurance $=10$.

Methods We used the National Cancer Database to identify cervical cancer patients diagnosed 2004-2017. Patients were classified by insurance status (Medicaid/uninsured vs. private) and hospitals were grouped into quartiles based on the proportion of uninsured/Medicaid patients (payer mix) (top quartile defined as SNHs). Quality-of-care was assessed by adherence to evidence-based metrics and survival by proportional hazards models. Individual contributions of insurance status and hospital payer mix on quality-of-care and survival were assessed.

Results A total of 124,339 patients including 11,338 uninsured (9.1\%) and 27,281 Medicaid (21.9\%) recipients treated at 1156 hospitals were identified. Quality-of-care was not significantly different across hospital quartiles. Adjusting for clinical/ demographic characteristics and hospital payer mix, treatment at a $\mathrm{SNH}$ was associated with a $14 \%$ higher mortality $(\mathrm{HR}=1.14 ; 95 \% \mathrm{CL}, 1.08-1.20)$ than Q1 hospitals. Adjusting for individual insurance, uninsured women had 32\% increased mortality $(\mathrm{HR}=1.32 ; 95 \% \mathrm{CI}, 1.26-1.38)$ and Medicaid recipients $40 \%$ increased $(\mathrm{HR}=1.40 ; 95 \% \mathrm{CI}, 1.35-1.44)$ compared to privately insured subjects. Adjusting for both payer mix and insurance status, only individual insurance retained a significant impact on mortality (table 1).

Conclusions Individual insurance status (having Medicaid or no insurance) may be a more important predictor of survival than site of care and hospital payer mix for women with cervical cancer.

\section{EPV040/\#185 THE PROGNOSTIC VALUE OF THE NUMBER OF POSITIVE LYMPH NODES AND THE LYMPH NODE RATIO IN EARLY STAGE CERVICAL CANCER}

${ }^{1} \mathrm{E}$ Olthof*, ${ }^{2} \mathrm{C}$ Mom, ${ }^{1} \mathrm{H}$ Wenzel, ${ }^{2} \mathrm{~J}$ Van Der Velden, ${ }^{1} \mathrm{M}$ Van Der Aa. ${ }^{1}$ Netherlands Comprehensive Cancer Organization, Research and Development, Utrecht, Netherlands; ${ }^{2}$ Amsterdam University Medical Centre, Gynaecological Oncology, Amsterdam, Netherlands

\subsection{6/ijgc-2021-IGCS.108}

Objectives Lymph node metastases are now incorporated into the 2018 International Federation of Gynecology and Obstetrics (FIGO) staging system for cervical cancer. However, the
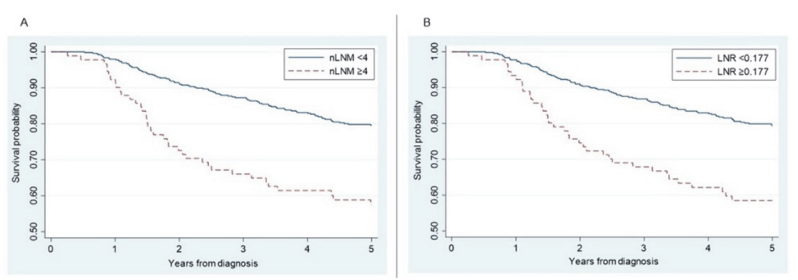

Abstract EPV040/\#185 Figure 1 Kaplan-Meier curves, 5-year overall survival categorizes by (A) nLNM risk-groups and (B) LNR risk-groups.

number of positive lymph nodes (nLNM) or the lymph node ratio (LNR) might provide a better prediction of survival. The aim of this study is to establish the impact of nLNM and LNR on survival in early-stage cervical cancer patients after surgery.

Methods In this population-based study, we selected all women diagnosed between 1995-2020 with FIGO 2009 stage IA2IIA1 cervical cancer and nodal metastases after radical hysterectomy and pelvic lymphadenectomy from the Netherlands Cancer Registry. Optimal cut-offs for prognostic stratification by $\mathrm{nLNM}$ and LNR were calculated to categorize patients in low- or high-risk groups. Kaplan-Meier overall survival analysis and flexible parametric relative survival analysis were used to determine the impact of nLNM and LNR on survival. Missing data were imputed.

Results Of 593 patients, 500 and 501 (84\%) were categorized in the low-risk and 93 and $92(16 \%)$ in the high-risk groups for nLNM $(\geq 4)$ and LNR $(\geq 0.177)$, respectively. Both highrisk groups had a worse 5 -year overall survival $(p<0.001)$ and were, together with non-squamous histology, independent risk factors for relative survival, with excess hazard ratios of 2.4 (95\% CI 1.6-3.5) for nLNM and 2.5 (95\% CI 1.7-3.8) for LNR.

Conclusions Presenting a patient's nodal status postoperatively by the number of positive nodes, or by its ratio, can support further risk stratification regarding survival in case of nodepositive early-stage cervical cancer.

\section{EPV041/\#196 HYSTERECTOMY AFTER CHEMORADIOTHERAPY FOR LOCALLY ADVANCED CERVICAL CANCER - EVALUATION OF PROGNOSTIC FACTORS AND SURVIVAL}

T Almeida De Sousa*, D Falcao Filho, M Amorim Nascimento, S Medeiros, R Oliveira Macedo, P Zanvettor. Aristides Maltez Hospital, Gynecologic Oncology, Salvador, Brazil

\subsection{6/ijgc-2021-IGCS.109}

Objectives Evaluate survival and prognostic factors of surgery after chemoradiotherapy (CRT) for locally advanced cervical cancer (LACC).

Methods A retrospective study was performed comparing patients who had undergone surgery following primary CRT for LACC to a control group treated only with CRT.

Results 176 patients fulfilled the inclusion criteria. Residual disease (RD) was found in $48(55,2 \%)$ patients submitted to surgery, $32(66,7 \%)$ had adenocarcinoma $(p=0,054)$. The main prognostic factor related to $\mathrm{RD}$ in a multivariate analysis was adenocarcinoma histologic type $(\mathrm{p}=0,005, \mathrm{HR}=5,54$ $(1,69-18,12))$. Patients with RD presented higher recurrence 